\title{
51. NUEVAS CITAS DE MACRÓFITOS ACUÁTICOS DE LAS ZONAS HÚME- DAS DE LA PROVINCIA DE MÁLAGA
}

\author{
José María NIETO CALDERA, Rafael Miguel CONDE-ÁLVAREZ, \\ María Angeles ARREBOLA BAUTISTA y Antonio FLORES-MOYA
}

New records of aquatic macrophytes from wetlands of the province of Málaga

Palabras clave. Lagunas salobres, macrófitos acuáticos, Málaga.

Key words. Aquatic macrophytes, Málaga.

Las abundantes precipitaciones que han tenido lugar durante otoño e invierno de los años 95-96 y 96-97 en el Sur de España, nos han movido a realizar una serie de muestreos en los humedales de la provincia de Málaga. Los lugares objetos de estudio han sido: desembocadura del Guadalhorce (Málaga), Laguna de Fuente de Piedra (Fuente de Piedra), Laguna de la Ratosa (Alameda), Laguna Serafina (Humilladero) y las lagunas del Cerero, Lobón, Marcela, Capacete, Dulce y Salada (Campillos).

Son numerosos y dispersos los estudios que hacen referencia a la composición florística de estas lagunas: Willkomm et Lange (1870), Reyes Prosper (1910), Corillion (1962), Guerlesquin (1963), Alonso et al. (1980), Asensi y Nieto Caldera (1981), Comelles (1982), Martínez Parras (1984), Gil y Molero Mesa (1984), Talavera et al. (1984), García Murillo y Talavera (1986), Guerra et al. (1986), Talavera et al. (1986), Cirujano et al. (1988), García Murillo (1989), Cirujano y García Murillo (1990a, b), Cirujano et al. (1992). En este trabajo aportamos seis nuevas citas de macrófitos acuáticos para la provincia de Málaga. Para la ordenación sistemática se han seguido los criterios de Valdés et al. (1987).

\section{NAJADACEAE}

\section{Najas marina L.}

MÁLAGA, desembocadura del Guadalhorce, 30SUF7059, R. M. Conde-Álvarez y J. M. Nieto Caldera, 4-6-97 (MGC 44946).

\section{POTAMOGETONACEAE}

\section{Potamogeton pectinatus L.}

CAMPILlOS, Laguna del Cerero, 30SUG3801, R. M. Conde-Álvarez y J. M. Nieto Caldera, 8-5-97 (MGC 44936). Laguna de la Marcela, 30SUF3698, R. M. CondeÁlvarez, J. M. Nieto Caldera, M. A. Arrebola Bautista y A. Flores-Moya, 25-7-97 (MGC 44944). Laguna Dulce, 30SUG3602, R. M. Conde-Álvarez y J. M. Nieto Caldera, 8-5-97 (MGC 44933).

MÁLAGA, desembocadura del Guadalhorce, 30SUF7059, R. M. Conde-Álvarez y J. M. Nieto Caldera, 4-6-97 (MGC 44935).

\section{RANUNCULACEAE}

Ranuculus peltatus Schrank subsp. fucoides (Freyn) Muñoz Garmendia

CAMPILlOS, Laguna Lobón, 30SUG3803, R. M. Conde-Álvarez y J. M. Nieto Caldera, 8-5-97 (MGC 44951). 


\section{RUPPIACEAE}

Ruppia drepanensis Tin ex Guss.

CAMPILLOS, Laguna Dulce, 30SUG3602, J. M. Nieto Caldera, 20-4-80 (MGC 6442).

\section{Ruppia maritima L.}

ALAMEDA, Laguna dela Ratosa, 30SUG4819, J. M. Nieto Caldera, 20-4-85 (MGC 8765).

HUMILLADERO, Laguna de la Serafina, 30SUG4716, J. M. Nieto Caldera y M. A. Arrebola Bautista, 8-5-97 (MGC 44941).

MÁLAGA, desembocadura del Guadalhorce, 30SUF7059, R. M. Conde-Álvarez y J. M. Nieto Caldera, 4-6-97 (MGC 44947).

Esta especie había sido citada anteriormente (sub Ruppia rostellata Koch) por Willkomm et Lange (1870:27) en las proximidades de Málaga. Nuestra cita confirma su presencia en este área.

\section{ZANNICHELLIACEAE}

Zannichellia obtusifolia Talavera, García Murillo y Smit

CAMPILLOS, Laguna del Cerero, 30SUG3801, R. M. Conde-Álvarez y J. M. Nieto Caldera, 25-7-97 (MGC 44938).

\section{BIBLIOGRAFÍA}

ALONSO, M., M. COMELLES y R. MARGALEF 1980- Nuevas citas de Althenia filiformis Petit. Lagascalia 9 (2): 220-223.

ASENSI, A. y J. M. NIETO CALDERA -1981Vegetación acuática, halófila y halonitrófila de la provincia de Málaga. Trabajos y monografías del Departamento de Botánica de Málaga 2: 105-122.

CIRUJANO, S. y P. J. GARCÍA MURILLO - 1990aMapa 435. Ruppia maritima L. var maritima, In J. Fernández Casas (ed.). Asientos para un atlas corológico de la flora occidental, 16. Fontqueria 28: 159-161.

CIRUJANO, S. y P. J. GARCÍA MURILLO - 1990bMapa 436. Ruppia drepanensis Tineo, In J. Fernández Casas (ed.). Asientos para un atlas corológico de la flora occidental, 16. Fontqueria 28: 161-163.
CIRUJANO, S., C. MONTES, P. MARTINO, S. ENRÍQUEZ y P. J. GARCÍA MURILLO -1988Contribución al estudio del género Riella Mont. (Sphaerocarpales, Riellaceae) en España. Limnetica 4: 41-50.

CIRUJANO, S., M. VELAYOS, F. CASTILla y M. GIL -1992- Criterios botánicos para la valoración de las lagunas y humedales españoles (Península Ibérica y las islas Baleares). ICONA. Madrid, $455 \mathrm{pp}$.

CORILLION, R. -1962- Contribution à l'étude des charophycées de la péninsule Ibérique et du Maroc occidental. Bulletín de la Société Scientifique de Bretagne 37: 65-80.

GARCIA MURILLO, P. J. y S. TALAVERA -1986- El género Althenia Petit. Lagascalia 14 (1): 102-114.

GIL, J. A. y J. MOLERO MESA -1984- Nueva cita de Riella helicophylla (Bory \& Mont.) Mont. para la Península Ibérica. An. Jard. Bot. Madrid 41 (1): 195-195.

GUERRA, J., E. RUIZ y C. SERGIO-1986- Sobre la distribución de Riella helicophylla (Bory et Mont.) Mont. en la Península Ibérica. Anales de Biología. Secciones de Biología Ambiental y Vegetal 11: 75-76.

MARTÍNEZ PARRAS, J. M. -1984- La vegetación lacustre de la depresión de Antequera. Collect. Bot. 15: 289-306.

REYES PROSPER, E. -1910- Las carófitas de España. Singularmente las que crecen en sus estepas. Ed. Imprenta Artística Española, Madrid, 206 pp.

TALAVERA, S., J. A. AMAT y A. FURES. -1984Althenia filiformis s.l. Lagascalia 12 (2): 252-253.

TALA VERA, S., P. GARCÍA MURILLO y H. SMIT -1986- Sobre el género Zannichellia L. (Zannichelliaceae), Lagascalia 14 (2): 220-223. VALDÉS, B., S. TALA VERA y E. FERNÁNDEZGALIANO - 1987- Flora Vascular de Andalucía Occidental.III. Ketres Editora S. A., Barcelona, $555 \mathrm{pp}$.

WILLKOMM, M. et J. LANGE -1870- Prodromus Florae Hispanicae. I. Stuttgartiae, 316 pp.

Aceptado para su publicación en Octubre 1997

Dirección de los autores. Departamento de Biología Vegetal. Facultad de Ciencias. Universidad de Málaga. Apdo. 59.29080 Málaga. E-mail: nieto@uma.es. 